\title{
Actividad de un "ensamblaje" de murciélagos en el norte de Brasil (Mammalia: Chiroptera)
}

\author{
Adrián Alonso Durán ${ }^{1,2^{*}} \&$ Neder Oviedo-Morales ${ }^{1,2}$ \\ 1. Grupo de Investigación en Zoología y Ecología, Universidad de Sucre. Cra 28 \# 5-267, Barrio Puerta Roja, Sincelejo, \\ Sucre, Colombia; adurandelaossa@gmail.com \\ 2. Centro de Estudos de Biodiversidade, Universidad Federal de Mato Grosso do Sul (UFMS). Avenida Costa e Silva, \\ s/n, Universitário, CEP 79070-900, Campo Grande, MS, Brasil; nedermur@gmail.com \\ * Correspondencia
}

Recibido 06-VI-2018. Corregido 18-X-2018. Aceptado 24-I-2019.

\begin{abstract}
Activity of a bat assemblage in northern Brazil (Mammalia: Chiroptera). The Amazon is considered one of the most biodiverse tropical ecosystems in the world thanks to the structural complexity that geology offers and the great biological richness of its soils. Further, concerning the Mammalian class, bats are one of the best represented taxa in the region, nevertheless their ecology is poorly studied. This study, evaluates the influence of environmental factors such as the climate season, temperature (maximum and minimum), relative humidity and lunar phase on the activity patterns (abundance and trophic guilds) of an assemblage of bats in the Brazilian central Amazon. This study was carry out in two municipalities of the state of Rondônia using eight mist nets during 62 nights of sampling, covering humid and dry periods. A total of 2499 individuals of 58 species were captured. The best represented family was the Phyllostomidae, while the species that recorded the highest frequency of captures was Carollia perspicillata $(\mathrm{N}=859)$, followed by C. brevicauda $(\mathrm{N}=209)$. There were no significant differences between the structure of the assemblage considering the climatic season (raindry). However, during the dry season a greater number of species and individuals was recorded ( 57 species, $\mathrm{N}=$ 1597 ) than in the rainy season ( 44 species, $N=902)$. The abundance of bats was influenced mainly by relative humidity $(\mathrm{P}=0.00)$ and minimal temperature $(\mathrm{P}=0.04)$, while the species responded differentially to the lunar phase. These results show the influence of environmental factors on the activity patterns of a bat assemblage in the Amazon, where the factors that influence them depends on the ecology of each species. However, studies carried out for longer periods are required to determine if these patterns are maintained over time.
\end{abstract}

Key words: Amazon; bats; climatic influence; lunar phase; Phyllostomidae.

Durán, A. A., \& Oviedo-Morales, N. (2019). Actividad de un "ensamblaje" de murciélagos en el norte de Brasil (Mammalia: Chiroptera). Revista de Biología Tropical, 67(1), 69-82.

El bosque del Amazonas es considerado el mayor y más productivo ecosistema terrestre del mundo y uno de los más biodiversos (Holdridge, 1967; Daly \& Prance, 1989; Hoorn \& Wesselingh, 2010). Característica atribuida a su complejidad estructural (August, 1983; Moreno \& Halffter, 2000) que deriva en diferencias edáficas, climáticas, topográficas y condiciones geológicas (Myster, 2009).
Los murciélagos representan el $24.8 \%$ de los mamíferos brasileros, ocupando el segundo lugar después de los roedores con $34.7 \%$ (Paglia et al., 2012). Son considerados como buenos modelos para estudiar los procesos ecológicos, debido a sus hábitos tróficos, alta riqueza, abundancia y a la relación que tienen con su ambiente (Bonnacorso, 1979; Kunz \& Fenton, 2003; Bernard, Tavares, \& Sampaio, 
2011; Bobrowiec, dos Santos, Gazarini, \& Haugaasen, 2014). En el Amazonas brasilero componen cerca del $87 \%$ de la quiropterofauna del país, destacando así la importancia de este bioma para la diversidad, no sólo de murciélagos, sino también de mamíferos en general (Bernard et al., 2011).

Se ha demostrado, para este taxón, que sus patrones de actividad son influenciados por variables ambientales, tales como: temperatura, humedad, precipitación, disponibilidad de luz, entre otros (Lang, Kalko, Rome, Bockholdt, \& Dechmann, 2006; Navara \& Nelson, 2007; Zarazúa-Carvajal, Saldaña-Vásquez, SandovalRuiz, Stoner, \& Benítez-Malvido, 2016). Estos pueden influenciar directamente en su riqueza, abundancia, patrones reproductivos, hábitos de comportamiento o forrajeo (Mello, Schittini, Seling, \& Bergallo, 2004; Saldaña-Vásquez \& Munguía-Rosas, 2013; Bobrowiec et al., 2014). En murciélagos, se ha registrado que su riqueza y abundancia están negativamente correlacionadas con la altitud y latitud (Stevens \& Willig, 2002; Bu et al., 2015), mientras que, la masa corporal se muestra influenciada por la temporada climática (Rughetti \& Toffoli, 2014).

Algunos estudios han evaluado la influencia de ciertas variables en el ensamblaje de este grupo taxonómico, como la influencia lunar y el tipo de ambiente (Mello et al., 2004; Esbérard, 2007; Saldaña-Vásquez \& MunguíaRosas, 2013; Bobrowiec et al., 2014; Heer, Helbig-Bonitz, Fernandes, Mello, \& Kalko, 2015). Mientras que, Grimshaw y Higgins (2017) encontraron que la diversidad filogenética en murciélagos mexicanos estuvo influenciada por la precipitación y la estacionalidad.

El conocimiento acerca de la ecología de murciélagos en la Amazonía, es aún escaso, sin embargo, en la actualidad se observa un aumento en estudios relacionados con los efectos de la fragmentación y el impacto de la regeneración del bosque en comunidades de murciélagos del Amazonas (Rocha et al., 2017; Farneda et al., 2018; Verde, Silva, \& Calouro, 2018), con lo cual se hace pertinente entender los patrones de actividad de las especies de este taxón debido a la importancia que cumplen en el mantenimiento de los ecosistemas, siendo controladores de insectos, polinizadores y dispersores de un gran número de semillas (Kalko, Handley, \& Handley, 1996; Galindo, 1998; Jones, Jacobs, Kunz, Willigs, \& Racey, 2009). Los objetivos del presente estudio fueron: 1) evaluar si la temporada climática (lluviosa y seca) tiene influencia en la composición específica de un ensamblaje de murciélagos en el Amazonas brasileño. Hipotetizamos que durante la temporada lluviosa el ensamblaje de murciélagos presentará una mayor diversidad, dado que las condiciones ambientales durante esta temporada les son más favorables y garantizaría una mejor disponibilidad de recursos (como ha sido encontrado por Pereira et al., 2009) y pretendemos evaluar si los patrones de actividad (riqueza, abundancia y gremios tróficos) de los murciélagos del ensamblaje en estudio responden a variables ambientales, tales como: temperatura, humedad relativa y porcentaje de fase iluminada de la luna, en la zona de estudio. La hipótesis planteada se sustenta en que la actividad de los murciélagos puede verse influenciada por variables ambientales, como la temperatura; cuando esta se presenta muy baja, su actividad disminuye (Kunz, 1982). Así mismo, se ha reportado comúnmente que en noches de luna llena las capturas de murciélagos son más bajas, respondiendo a un fenómeno conocido como "fobia lunar" (Morrison, 1978), por consiguiente, es de esperar que en el presente estudio sus abundancias sean influenciadas por las variables ambientales mencionadas.

\section{MATERIALES Y MÉTODOS}

Área de estudio: El área de estudio comprende los municipios de Porto Velho y Jaci Paraná en el estado de Rondônia (norte de Brasil), ubicados a la margen del rio Madeira en la región del Amazonas (Apéndice digital 1). Este rio se constituye como uno de los mayores tributarios del rio Amazonas, siendo responsable cerca del $15 \%$ de su volumen (Goulding, Barthem, \& Ferreira, 2003). Presenta una precipitación media anual de 1700-2000 mm, 
un período de alta precipitación entre octubre y abril, y un período de baja precipitación de mayo a septiembre.

La vegetación es del tipo bosque tropical denso, con mosaicos de bosques de tierra firme e inundable (Veloso, Rangel, \& Lima, 1991) y predominio de bosque abierto ombrófilo en el que, además, se diferencian tres subtipos: con palmas (es el más común), sorocas (Phenakospermum guyannense) y bambú. Su formación data del Plioceno-Pleistoceno $(<3.6$ millones de años), con recientes depósitos fluviales (< 10000 años; RADAMBRASIL, 1978). El área de estudio se está viendo fuertemente afectada por el crecimiento urbano, construcción de vías y principalmente por la construcción de la presa hidroeléctrica Jirau (Perigolo, Medeiros, \& Simon, 2017).

Trabajo de campo: El estudio se llevó a cabo de junio 2010 a septiembre 2011 abarcando 62 noches de muestreo durante los períodos de mayor y menor precipitación.

Para la captura de murciélagos se utilizaron ocho redes de niebla de $12 \times 2.4 \mathrm{~m}$ abiertas a nivel de sotobosque desde las 18:0000:00 hrs, con revisiones cada $30 \mathrm{~min}$. Los individuos capturados eran transportados en sacos de tela hasta el campamento, donde fueron medidos (antebrazo, pata, oreja y trago), pesados, sexados e identificados mediante las claves dicotómicas de Lim y Engstrom (2001) y con ayuda de descripciones taxonómicas de Simmons (1996), Simmons y Voss (1998), Charles-Dominique, Brosset y Jouard (2001) y Gardner (2007) para finalmente ser liberados. Los individuos sobre los que aún se tenían dudas acerca de su identificación fueron colectados y comparados con especímenes de la colección de mamíferos del Instituto Nacional de Pesquisas Amazónicas (INPA), donde además fueron depositados (códigos: INPA 60256273), y posteriormente fueron confirmados mediante la secuenciación del gen cyt $\mathrm{b}$. Para la nomenclatura taxonómica se siguió a Simmons (2005), mientras que para la asignación de gremios tróficos se siguió a Kalko et al. (1996) y Rojas, Vale, Ferrero y Navarro (2011).
Análisis de datos: Para evaluar la completitud en los muestreos se realizó una curva de acumulación de especies empleando el estimador de riqueza Chao 1 (Colwell \& Coddintong, 1994; Moreno \& Halffer, 2000; Gotelli \& Colwell, 2001), en el que, además, se calculó la diversidad verdadera para ambas temporadas climáticas (lluviosa y seca), mediante la función "ggiNEXT" del paquete iNEXT (Hsieh, $\mathrm{Ma}$, \& Chao, 2016) para la plataforma $\mathrm{R}(\mathrm{R}$ Core Team, 2014). Los patrones de actividad de los murciélagos fueron evaluados en términos de su riqueza, abundancia y gremios tróficos.

Se calculó la equitatividad de Pielou con el fin de determinar si existe homogeneidad en las abundancias de las especies de murciélagos del área de estudio (Moreno, 2001). Las diversidades (en temporada lluviosa y seca) calculadas anteriormente fueron comparadas mediante la prueba t de diversidad (Zar, 1996; Moreno, Barragán, Pineda, \& Pavón, 2011). Estos análisis se realizaron en el software PAST v 3.12 (Hammer \& Harper, 2016).

Para evaluar la composición específica (riqueza) en el ensamblaje de murciélagos, de acuerdo a la temporada climática (lluviosa y seca), se realizó una Ordenación por Escalonamiento Multidimensional no Métrico (NMDS) utilizando la función "isoMDS" del paquete MASS (Venables \& Ripley, 2002), empleando el índice de Jaccard (Legendre \& Legendre, 2012), en la plataforma R (R Core Team, 2014). Fue realizada una PERMANOVA para determinar diferencias estadísticamente significativas entre las comparaciones del ensamblaje durante cada temporada.

Para evaluar la influencia que tienen la temperatura $\left({ }^{\circ} \mathrm{C}\right.$ ) (temperatura máxima y temperatura mínima) y la humedad relativa (\%) sobre las abundancias de los murciélagos (incluyendo todas las especies del ensamblaje) se solicitaron los datos para el 2010 y 2011 a la Estación Automática de Porto Velho (Rondónia) (Porto Velho-A925, 845' S \& $\left.63^{\circ} 28^{\prime} \mathrm{W}, 95 \mathrm{msnm}\right)$ del Instituto Nacional de Meteorología (INMET); sin embargo, solo estaban disponibles datos de junio, julio, septiembre y diciembre 2010 y abril y mayo 2011. 
Adicionalmente, contaban únicamente con datos de temperatura $\left({ }^{\circ} \mathrm{C}\right)$ y humedad relativa (\%) debido a fallas en la estación (por eso no se consideró la precipitación como variable continua). Para los datos de temporada climática, se organizaron las fechas de colectas de acuerdo a la respectiva temporada (lluviosa y seca). Con estas variables se realizaron Modelos Lineales Generalizados (GLM, por sus siglas en inglés), con la distribución binomial negativa (Zur, Ieno, Walker, Saveliev, \& Smith, 2009), mediante la función "gam" del paquete mgcv para R (Wood, 2014), con el fin de evaluar su influencia en la abundancia de murciélagos.

Finalmente, para analizar si el ciclo lunar estaba relacionado con las abundancias de las especies de murciélagos de acuerdo al gremio trófico (Kalko et al., 1996, Rojas et al., 2011), se calculó el porcentaje de fase iluminada de la Luna por cada noche de muestreo (Esbérard, 2007), mediante la utilización del software Moontool v. 2.0 (Walker, 1999). Con estos datos y una matriz de abundancias se realizó un gráfico compuesto, propuesto por Victor Lemes Landeiro, mediante la función "genérico" para la plataforma R (R Core Team, 2014), en el cual se puede visualizar de manera condensada y bastante simple, como se distribuyen las abundancias de las especies a través de un gradiente ambiental (porcentaje de fase iluminada de la Luna, en este caso). En este tipo de gráficos se excluyó el gremio de los hematófagos debido a la poca representatividad de especies. Fue utilizada la correlación de Kendall con el fin de determinar la significancia de las relaciones (Zar, 1996) (Apéndice digital 2).

\section{RESULTADOS}

Fueron capturados 2499 individuos pertenecientes a 58 especies, 36 géneros y seis familias. Phyllostomidae fue la familia que registró el mayor número de especies y la mayor frecuencia de capturas (49 especies, $96.96 \%$ ) (Cuadro 1). Carollia perspicillata (Linnaeus, 1758) $(\mathrm{N}=859)$, Carollia brevicauda (Schinz,

CUADRO 1

Composición de especies del ensamblaje de murciélagos por temporada climática (lluviosa y seca) en la Amazonía brasileña

TABLE 1

Composition of species in the assemblage of bats by climatic season (wet and dry) in the Brazilian Amazon

\begin{tabular}{|c|c|c|c|c|c|}
\hline \multirow{2}{*}{ Familia } & \multirow{2}{*}{ Subfamilia } & \multirow{2}{*}{ Especie } & \multicolumn{2}{|c|}{ Temporada climática } & \multirow{2}{*}{ TOTAL } \\
\hline & & & Lluvia & Seca & \\
\hline \multirow[t]{3}{*}{ Emballonuridae } & & Rhynchonycteris naso (Wied-Neuwied 1820) & 0 & 2 & 2 \\
\hline & & Saccopteryx bilineata $($ Temminck 1838$)$ & 2 & 1 & 3 \\
\hline & & Saccopteryx leptura (Schreber 1774) & 3 & 4 & 7 \\
\hline \multirow[t]{13}{*}{ Phyllostomidae } & Carollinae & Carollia benkeithi Solari y Baker 2006 & 3 & 10 & 13 \\
\hline & & Carollia brevicauda $($ Schinz 1821) & 96 & 113 & 209 \\
\hline & & Carollia perspicillata (Linnaeus 1758) & 347 & 512 & 859 \\
\hline & & Rhynophylla fischerae Carter 1966 & 1 & 6 & 7 \\
\hline & & Rhynophylla pumilio Peters 1865 & 71 & 114 & 185 \\
\hline & Desmodontinae & Desmodus rotundus (É. Geoffroy 1810) & 1 & 4 & 5 \\
\hline & & Diphylla ecaudata Spix 1823 & 1 & 1 & 2 \\
\hline & Glossophaginae & Choeroniscus minor (Peters 1868) & 2 & 1 & 3 \\
\hline & & Glossophaga longirostris Miller 1898 & 1 & 0 & 1 \\
\hline & & Glossophaga soricina (Pallas 1776) & 6 & 10 & 16 \\
\hline & & Lichonycteris degener (Miller 1831)* & 0 & 1 & 1 \\
\hline & & Lonchophylla thomasi Allen 1904 & 21 & 43 & 64 \\
\hline & & Lyonycteris spurrelli Thomas 1913* & 0 & 1 & 1 \\
\hline
\end{tabular}


CUADRO 1 (Continuación) / TABLE 1 (Continued)

\begin{tabular}{|c|c|c|c|c|c|}
\hline \multirow{2}{*}{ Familia } & \multirow{2}{*}{ Subfamilia } & \multirow{2}{*}{ Especie } & \multicolumn{2}{|c|}{ Temporada climática } & \multirow{2}{*}{ TOTAL } \\
\hline & & & Lluvia & Seca & \\
\hline & \multirow[t]{19}{*}{ Phyllostominae } & Chrotopterus auritus (Peters 1856)* & 6 & 7 & 13 \\
\hline & & Glyphonycteris daviesi (Hill 1964) & 0 & 2 & 2 \\
\hline & & Glyphonycteris silvestris Thomas $1896^{*}$ & 1 & 3 & 4 \\
\hline & & Lampronycteris brachyotis (Dobson 1879)* & 1 & 4 & 5 \\
\hline & & Lophostoma brasiliense (Peters 1866)* & 6 & 2 & 8 \\
\hline & & Lophostoma silvicolum d'Orbigny 1836 & 48 & 50 & 98 \\
\hline & & Micronycteris hirsuta (Peters 1869)* & 0 & 3 & 3 \\
\hline & & Micronycteris megalotis (Gray 1842) & 4 & 3 & 7 \\
\hline & & Micronycteris microstis Miller 1898* & 5 & 4 & 9 \\
\hline & & Micronycteris schmidtorum Sanborn 1935* & 0 & 1 & 1 \\
\hline & & Mimon bennettii (Gray 1838)* & 0 & 1 & 1 \\
\hline & & Mimon crenulatum (É. Geoffroy 1810) & 7 & 6 & 13 \\
\hline & & Phylloderma stenops Peters 1865 & 3 & 8 & 11 \\
\hline & & Phyllostomus discolor Wagner 1843* & 2 & 5 & 7 \\
\hline & & Phyllostomus elongatus (É. Geoffroy 1810) & 22 & 46 & 68 \\
\hline & & Phyllostomus hastatus (Pallas 1767) & 4 & 5 & 9 \\
\hline & & Tonatia saurophila Koopman y Williams 1951* & 26 & 19 & 45 \\
\hline & & Trachops cirrhosus (Spix 1823) & 22 & 18 & 40 \\
\hline & & Trinycteris nicefori Sanborn 1949* & 14 & 18 & 32 \\
\hline & \multirow[t]{17}{*}{ Stenodermatinae } & Artibeus concolor Peters 1865 & 0 & 4 & 4 \\
\hline & & Artibeus lituratus (Olfers 1818) & 16 & 103 & 119 \\
\hline & & Artibeus obscurus Schinz 1821 & 46 & 112 & 158 \\
\hline & & Artibeus planirostris (Spix 1823) & 40 & 134 & 174 \\
\hline & & Chiroderma villosum Peters 1860 & 0 & 2 & 2 \\
\hline & & Dermanura anderseni (Osgood 1916) & 0 & 3 & 3 \\
\hline & & Dermanura cinerea Gervais 1856 & 0 & 32 & 32 \\
\hline & & Dermanura gnoma (Handley 1987) & 8 & 29 & 37 \\
\hline & & Mesophylla macconnellii Thomas 1901 & 5 & 29 & 34 \\
\hline & & Platyrrhinus brachycephalus (Rouk y Carter 1972) & 1 & 2 & 3 \\
\hline & & Platyrrhinus incarum (Thomas 1912) & 4 & 7 & 11 \\
\hline & & Sturnira lilium (É. Geoffroy 1810) & 2 & 5 & 7 \\
\hline & & Sturnira tildae de la Torre 1959 & 12 & 13 & 25 \\
\hline & & Uroderma bilobatum Peters 1866 & 11 & 26 & 37 \\
\hline & & Uroderma magnirostrum Davis 1968* & 2 & 2 & 4 \\
\hline & & Vampyressa thyone Thomas 1909 & 0 & 1 & 1 \\
\hline & & Vampyriscus bidens (Dobson 1878) & 8 & 22 & 30 \\
\hline Natalidae & & Natalus spiritosantensis Ruschi $1951^{*}$ & 0 & 1 & 1 \\
\hline Mormoopidae & & Pteronotus parnellii Gray 1843 & 15 & 30 & 45 \\
\hline \multirow[t]{2}{*}{ Vespertilionidae } & & Myotis nigricans (Schinz 1821)* & 1 & 6 & 7 \\
\hline & & Myotis riparius Handley $1960^{*}$ & 2 & 2 & 4 \\
\hline \multirow[t]{2}{*}{ Thyropteridae } & & Thyroptera discifera (Lichtenstein y Peters 1855) & 0 & 1 & 1 \\
\hline & & Thyroptera tricolor Spix 1823* & 3 & 3 & 6 \\
\hline Total & & 58 & 902 & 1597 & 2499 \\
\hline
\end{tabular}

* Nuevos registros para el estado de Rondónia. / * New records for the state of Rondónia. 
1821) ( $\mathrm{N}=209)$ y Rhynophylla pumilio (Peters, 1865) $(\mathrm{N}=185)$, fueron los murciélagos más capturados, todos pertenecientes a la subfamilia Carollinae (Phyllostomidae).

Durante la temporada lluviosa se registró un índice de Shannon menor $(\mathrm{H}=2.51)$ que en la temporada seca $(H=2.73)$, sin embargo, la prueba $t$ de diversidad, no encontró significancia en cuanto a diferencias entre estos valores $(\mathrm{t}$ $=0.76, \mathrm{P}=0.44)$. El mayor número de especies se presentó durante la temporada seca (57 especies), en contraste con la temporada lluviosa (44 especies). El valor en el índice de equidad fue similar para ambas temporadas (lluviosa: J $=0.66$, seca: $\mathrm{J}=0.67$ ).

Al evaluar la completitud entre los muestreos, se observa que durante ambas temporadas climáticas es alcanzada la asíntota en la curva de acumulación de especies (Fig. 1), corroborado con una buena eficiencia de muestreos para ambas (lluviosa $=89.20 \%$, seca $=88.47 \%$ ).

El análisis NMDS y la PERMANOVA indican que la composición de especies no se encuentra influenciada por esta variable ( $\mathrm{F}=$ 1.57, $\mathrm{P}=0.08$ ) (Fig. 2). Mientras que, según

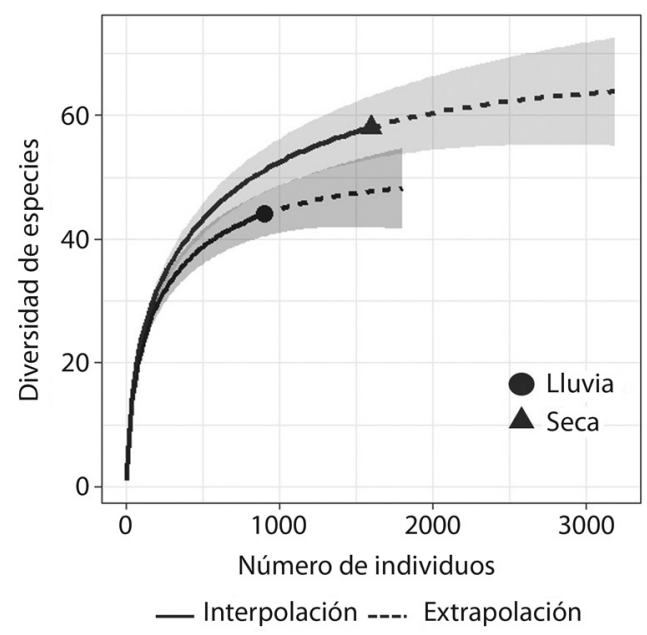

Fig. 1. Curva de acumulación de especies para cada temporada climática en el área de estudio de la Amazonía brasilera. El área sombreada corresponde al nivel de confianza en un $95 \%$.

Fig. 1. Species accumulation curve for each climate season in the study area of the Brazilian Amazon. The shaded area corresponds to the $95 \%$ confidence level. los resultados de los GLM's, el mejor modelo propuesto que mejor explica la influencia de variables ambientales sobre la abundancia de murciélagos, es el modelo 1 (M1, Cuadro 2), en donde existe una relación significativa entre las abundancias y las variables: temperatura mínima $(\mathrm{P}=0.04)$ y humedad relativa $(\mathrm{P}=0.00)$. Sin embargo, la primera se encuentra negativamente relacionada con las abundancias, como lo sugiere el signo negativo en el coeficiente $\beta$ del modelo, a diferencia de la humedad relativa (Apéndice digital 3).

Por otro lado, no se evidencia relación estadísticamente significativa entre el porcentaje de fase iluminada de la Luna y las abundancias de las especies de acuerdo a su gremio trófico, sin embargo, se puede observar de manera individual, que, en los insectívoros aéreos de espacio altamente cerrado, la especie Pteronotus parnelli (Gray, 1843) presentó mayores abundancias en días con menor porcentaje de fase iluminada de la Luna (Fig. 3A). Este mismo patrón se puede observar en algunas especies del gremio de los frugívoros aéreos de espacio altamente cerrado: Sturnira tildae (de la Torre, 1959), Artibeus obscurus (Schinz, 1821), C. perspicillata y C. brevicauda (Fig. 3C), así como en Lonchophylla thomasi (Allen,

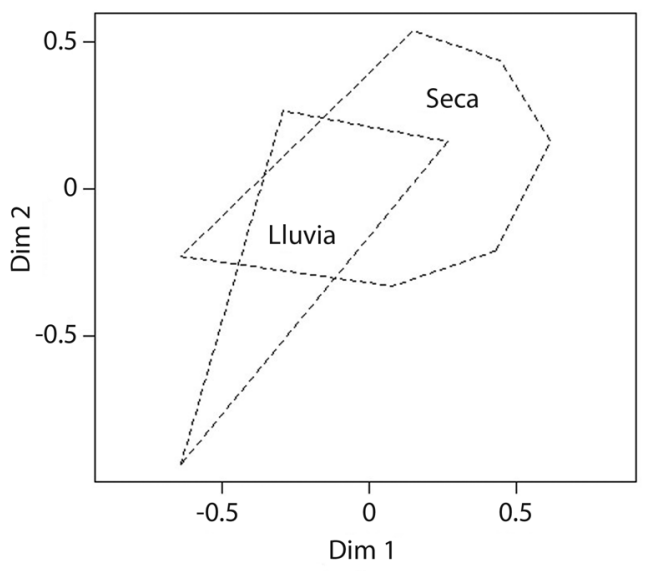

Fig. 2. Ordenación por Escalonamiento Multidimensional no Métrico

del ensamblaje de murciélagos en la zona de estudio.

Fig. 2. Non-Metric Multidimensional Scaling of the assembly of bats in the study area. 
CUADRO 2

Modelos Lineales Generalizados para evaluar la influencia de variables ambientales sobre las abundancias de las especies del ensamblaje de murciélagos en la zona de estudio

TABLE 2

Generalized Linear Models to evaluate the influence of environmental variables on the abundances of bat species in the study area

\begin{tabular}{|c|c|c|c|c|c|}
\hline $\begin{array}{c}\text { Variable respuesta } \\
=\text { Abundancia }\end{array}$ & Variables descriptoras del modelo & $\mathrm{R}^{2}$ & $\mathrm{AICc}$ & $\Delta_{\mathrm{i}}$ & $\mathrm{W}_{\mathrm{i}}$ \\
\hline M1 & Temperatura mínima*+Humedad relativa $* *$ & 0.19 & 271.5 & 0.00 & 0.33 \\
\hline M2 & Humedad relativa+Período de precipitación & 0.17 & 272.5 & 1.01 & 0.20 \\
\hline M3 & Temperatura mínima+Humedad relativa+Período de precipitación & 0.21 & 273.7 & 2.17 & 0.11 \\
\hline M4 & $\begin{array}{l}\text { Temperatura máxima+Temperatura mínima+Período de } \\
\text { precipitación }\end{array}$ & 0.20 & 274.0 & 2.48 & 0.09 \\
\hline M5 & Temperatura máxima+Temperatura mínima*+Humedad relativa & 0.20 & 274.0 & 2.54 & 0.09 \\
\hline M6 & Temperatura máxima + Humedad relativa + Período de precipitación & 0.20 & 274.2 & 2.74 & 0.08 \\
\hline M7 & Temperatura mínima+Período de precipitación & 0.11 & 274.3 & 2.84 & 0.08 \\
\hline M8 & $\begin{array}{l}\text { Temperatura máxima+Temperatura mínima+Humedad } \\
\text { relativa+Período de precipitación }\end{array}$ & 0.21 & 276.8 & 5.33 & 0.02 \\
\hline
\end{tabular}

$\mathrm{AICc}=$ Criterio de Información Akaike ajustado a pequeñas muestras (Anderson, 2008), $\Delta_{i}=$ Delta AIC, $\mathrm{W}_{i}=$ Peso AIC. * $=$ Nivel de significancia $0.05, * *=$ Nivel de significancia 0.001 .

$\mathrm{AICc}=$ Akaike Information Criterium adjusted to small samples (Anderson, 2008), $\Delta \mathrm{i}=$ Delta AIC, Wi $=$ AIC Weight. $*=$ Level of significance $0.05, * *=$ Level of significance 0.001 .

1904) (nectarívoro de espacio altamente cerrado, Fig. 3F). Las abundancias de las especies restantes no se mostraron influenciadas por la luminosidad de la Luna (Apéndice digital 2, Apéndice digital 4).

\section{DISCUSIÓN}

Las 54 especies de murciélagos listadas en el presente documento corresponden al $36.98 \%$ del total registrado para la Amazonía brasilera, reportando 18 nuevas especies para el estado de Rondônia (Cuadro 1) (Bernard et al., 2011).

La familia con el mayor número de especies fue Phyllostomidae, un resultado habitual en los inventarios de murciélagos en el Neotrópico (Brosset \& Charles-Dominique, 1990; Bernard \& Fenton, 2002; Durán \& Canchila, 2015), determinado probablemente por el método de captura (redes en sotobosque). Ubicar redes a nivel de dosel presenta muchas dificultades en campo, generando así un sesgo en los resultados, debido a que la mayoría de las especies de la familias Vespertilionidae, Molossidae y Emballonuridae forrajean en el estrato más alto del bosque (Fleming, Hooper, \& Wilson, 1972; Gardner, 2007); sin embargo, es desconocido hasta qué punto esto tiene influencia en los resultados, por este motivo se consideraron todas las familias en este estudio.

Los filostómidos son bien conocidos por abarcar un amplio rango de gremios tróficos (Bonnaccorso, 1979; Stoner, 2005), así como por su alta plasticidad (en la mayoría de las especies que componen esta familia) para adaptarse a los ambientes intervenidos (Fleming, 1986; Freeman, 2000). En el área de estudio, al presentar una creciente intervención antrópica (Fearnside, 2014), esta familia siempre dominará los registros de murciélagos (Stoner, 2005; Gardner, 2007). Dicho taxón se encuentra fuertemente dominado por la especie $C$. perspicillata, lo cual ya ha sido reportado por otros autores en la misma región (Bernard \& Fenton, 2002; Cruz, Martínez, \& Fernandes, 2007; Pereira, Marques, \& Palmeirim, 2010). 

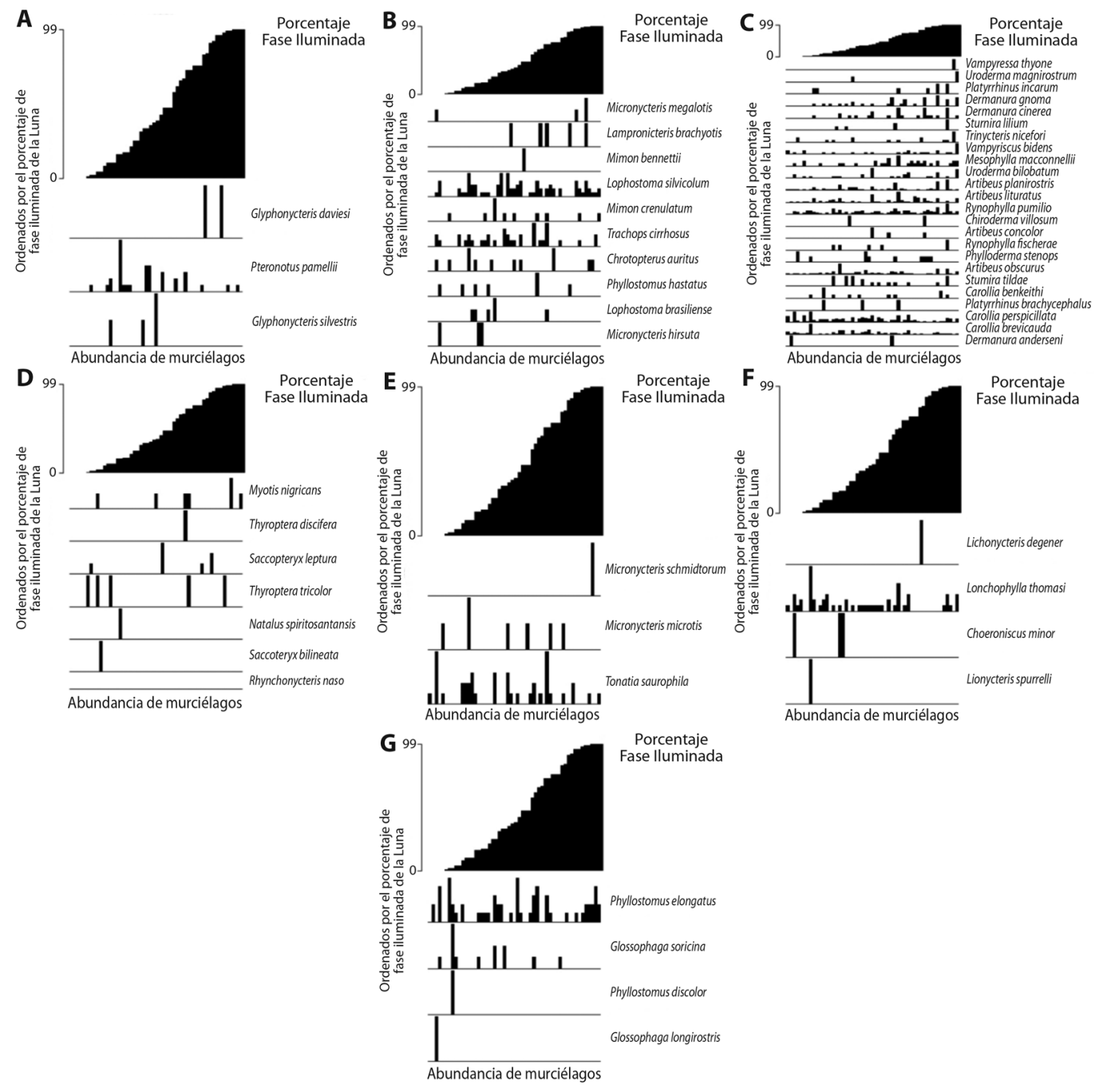

Fig. 3. Gráfico compuesto cuyas especies están ordenadas de acuerdo con el porcentaje de fase iluminada de la Luna. A. Insectívoros aéreos de espacio altamente cerrado, B. Carnívoros de espacio altamente cerrado, C. Frugívoros aéreos de espacio altamente cerrado, D. Insectívoros aéreos de espacio cerrado, E. Insectívoros de presas posadas en espacio altamente cerrado, F. Nectarívoros de espacio altamente cerrado, G. Omnívoros.

Fig. 3. Composite graph whose species are arranged according to the percentage of illuminated phase of the Moon. A. Highly cluttered space/aerial insectivore, B. Highly cluttered space/gleaning carnivore, C. Highly cluttered space/gleaning frugivore, D. Cluttered space/aerial insectivore, E. Highly cluttered space/gleaning insectivore, F. Highly cluttered space/ gleaning nectarivore, G. Omnivores.

Además de abundante, es un murciélago muy común en los bosques de la Amazonía (Bernard et al., 2011), siendo considerada una especie de hábitos más generalistas en cuanto a dieta y comportamiento, al igual que su congénere, C. brevicauda (Bonaccorso, 1976; Fleming, 1986).
Estudios previos en otros ecosistemas, como en el Bosque Atlántico en Brasil (Gomes, Pires, Martins, Lourenço, \& Peracchi, 2015), han demostrado que la temporada climática tiene una importante influencia en la diversidad de los ensamblajes de murciélagos, en contraposición con lo encontrado por Zarazúa-Carvajal 
et al. (2016), en un bosque seco tropical en México y Bobrowiec et al. (2014) en la Amazonía central brasilera, así como en el presente estudio. Si bien, la disponibilidad de recursos alimenticios está determinada por el factor clima, como ya ha sido encontrado por Pereira et al. (2009) en la zona de estudio, hay un excedente de frutos que no es consumido durante la temporada lluviosa, donde son alcanzados los mayores picos de fructificación, como es expuesto por Bobrowiec et al. (2014), con lo cual y según lo hallado por Pereira et al. (2009), en cualquier época del año se presentaría una importante oferta trófica (frutos) que sustentaría las poblaciones de murciélagos frugívoros. Considerando que este gremio es el dominante (Cuadro 1 y Apéndice digital 2), la estructura del ensamblaje no se vería significativamente influenciado por el clima, como se evidencia en las métricas de diversidad, siendo similares para ambas temporadas. Sin embargo, este excedente de frutas puede ser mejor aprovechado por algunas especies del gremio de los frugívoros (Bobrowiec et al., 2014), mostrando mayores abundancias en el período seco (Cuadro 1 y Apéndice digital 2), lo que contribuye en aumentar el total de murciélagos durante esta temporada climática; esto explicaría la marcada abundancia diferencial por temporada (lluviosa y seca), mientras que la diversidad es similar, ya que la composición de especie no cambia significativamente, como es demostrado en el análisis NMDS.

En este estudio, además de la temporada climática también se consideraron otras variables ambientales, como la temperatura, mostrando una relación negativa respecto a la abundancia de murciélagos. El mayor número de especies registradas se encuentran entre los 30-33 ${ }^{\circ} \mathrm{C}$ aparentando ser un rango de temperatura más óptimo para la actividad de murciélagos en la Amazonía central brasilera. Estos mamíferos son diferentes de otros heterotermos debidos que su temperatura de reposo y su tasa metabólica dependen de la temperatura del ambiente, lo cual se debe a su falta de termorregulación (Hock, 1951; Kunz, 1982). Sin embargo, se requieren de estudios más detallados para evaluar si este patrón se mantiene. Mientras que, la humedad relativa fue positivamente relacionada con la abundancia de murciélagos, siendo concordante con lo hallado por Lacki (1984) en la especie Myotis lucifugus (Le Conte, 1831) e influenciado probablemente por una alta actividad de insectos (Robinet \& Roques, 2010), considerando que esta especie es insectívora. Como ya se ha mencionado anteriormente, los filostómidos son especies que abarcan un amplio rango de gremios tróficos, siendo principalmente frugívoros e insectívoros (Bonnacorso, 1979; Kalko et al., 1996; Gardner, 2007), con lo cual al aumentar la oferta trófica aumentarían sus patrones de actividad, ya que estos pueden variar en forma diaria o estacional en respuesta a una variedad de factores exógenos y endógenos, incluida la abundancia de insectos (Hayes, 1997).

El porcentaje de fase iluminada de la Luna es una variable muy interesante de medir. Se ha registrado que ésta reprime la actividad en muchas especies animales incluyendo crustáceos (Wolcott \& Wolcott, 1982), insectos (Williams \& Singh, 1951), pequeños mamíferos (Clarke, 1983), entre otros. Una de las razones es que, un incremento en la intensidad de luz durante la noche aumentaría el riesgo en la depredación, por lo que la disponibilidad de presas se reduce (Brigham \& Barclay, 1992; Lang et al., 2006). La figura 3 muestra la influencia de esta variable en la abundancia de murciélagos, agrupados por gremios tróficos, en la que no se evidencia una clara relación, ya que la mayoría de las especies son registradas en cualquier porcentaje de luminosidad, en concordancia con lo reportado por Esbérard (2007). En los insectívoros, en cambio, las especies con mayor número de capturas mostraron patrones en respuesta a la luminosidad de la Luna, como en: $P$. parnelli (Fig. 3A, insectívoros aéreos de espacio altamente cerrado), Saccopterix leptura (Schreber, 1774) (Fig. 3D, insectívoros aéreos de espacio cerrado), Micronycteris microtis (Miller, 1898) y Tonatia saurophila (Koopman \& Williams, 1951) (Fig. 3E, insectívoros de presas posadas en espacio altamente cerrado), sin embargo, en 
la mayoría no se evidenció una respuesta de sus abundancias en función de esta variable, muy probablemente debido al bajo número de capturas en muchas de las especies registradas en este estudio, aun así, una influencia diferencial en la actividad de murciélagos puede deberse a la disponibilidad de sus presas más habituales, como fue encontrado por Lang et al. (2006) en Lophostoma silvicolum (d'Orbigny, 1836) y Börk (2006) en Noctilio leporinus (Linnaeus, 1758), este último se registró más activo en noches cuando la Luna estaba menos iluminada. En los gremios restantes se presentó un comportamiento similar.

En un meta-análisis respecto al tema, realizado por Saldaña-Vázquez y Munguía-Rosas (2013), se reportaron evidencias demostrando que, dependiendo de la latitud, el efecto de la influencia lunar era más fuerte (por ejemplo, en los trópicos), de la misma manera la actividad de los murciélagos en términos de hábitos de forrajeo, se ve afectada por la luminosidad de la Luna. Los murciélagos que forrajean en el dosel se mostraron negativamente influenciados por ésta variable, mientras que Gutiérrez et al. (2014) encontraron que A. planirostris (Spix, 1823) detectaba mejor su alimento en noches muy luminosas. Así mismo, en Appel, López-Baucell, Magnusson y Bobrowiec (2017), P. parnellii se mostró más activo en noches de Luna llena, a diferencia de $M$. riparius (Handley, 1960). En este mismo estudio otras especies como $S$. bilineata (Temminck, 1838) y Cormura brevirostris (Wagner, 1843) no se vieron influenciados.

En conclusión, respecto a este tema, aún sigue siendo motivo de un amplio debate, con estudios que reportan evidencias a favor (Morrison, 1978; Börk, 2006, Lang et al., 2006) y otros en contra (Karlsson, Eklöf, \& Rydell, 2002; Thies, Kalko, \& Schnitzler, 2006), con lo cual se requieren de diseños muestreales más específicos y a más largo plazo para así encontrar patrones más definidos que demuestren la influencia diferencial de la luminosidad en los murciélagos.
Declaración de ética: los autores declaran que todos están de acuerdo con esta publicación y que han hecho aportes que justifican su autoría; que no hay conflicto de interés de cualquier tipo; y que han cumplido con todos los requisitos y procedimientos éticos y legales pertinentes. El documento firmado se encuentra en los archivos de la revista.

\section{AGRADECIMIENTOS}

Los autores ofrecen sus agradecimientos al Instituto Nacional de Pesquisas Amazônicas (INPA), Manaus (Brasil) por los datos proporcionados para la realización del estudio, al Conselho Nacional de Desenvolvimento Científico e Tecnológico (CNPq) por la concesión de las becas para la realización de la maestría (proceso 131544/2016-0), así como a Marcelo Isidro Figueroa por la ayuda en los análisis estadísticos y a Silvia Cassinera por la revisión del resumen en inglés.

\section{RESUMEN}

La Amazonía es uno de los ecosistemas tropicales más biodiversos del mundo, característica atribuida a la complejidad estructural que le brinda la geología y la gran riqueza de sus suelos. Los murciélagos constituyen uno de los taxones de la clase Mammalia mejor representados en esta región, aun así, existen claros vacíos de conocimiento, principalmente, respecto a su ecología, con lo cual, en el presente estudio se pretende evaluar la influencia que tienen factores ambientales, como la temporada climática, temperatura (máxima y mínima), humedad relativa y porcentaje de fase iluminada de la Luna, en los patrones de actividad (riqueza, abundancia y gremios tróficos) de un ensamblaje de murciélagos en la Amazonía central brasilera. El estudio fue llevado a cabo en dos municipios del estado de Rondônia, empleando ocho redes de niebla, durante 62 noches de muestreo, abarcando las temporadas lluviosa y seca. En total fueron capturados 2499 individuos de 58 especies, la familia mejor representada fueron los Phyllostomidae, mientras que la especie que registró la mayor frecuencia de capturas fue Carollia perspicillata $(\mathrm{N}=859)$, seguida de $C$. brevicauda $(\mathrm{N}=209)$. No se presentaron diferencias entre la estructura del ensamblaje considerando la temporada climática (lluviosa-seca), sin embargo, durante la temporada seca se registró un mayor número de especies e individuos 
(57 especies, $\mathrm{N}=1597$ ), que en la temporada lluviosa (44 especies, $\mathrm{N}=902$ ). La abundancia de murciélagos estuvo influenciada principalmente, por la humedad relativa $(\mathrm{P}=$ $0.00)$ y la temperatura (mínima) $(\mathrm{P}=0.04)$, mientras que, las especies respondieron diferencialmente al porcentaje de fase iluminada de la luna. En estos resultados se evidencia la influencia de factores ambientales en los patrones de actividad del ensamblaje de murciélagos en la Amazonía, en donde la ecología de las especies determina el tipo de influencia. Sin embargo, se requieren de estudios llevados a cabo por períodos más prolongados, para determinar si estos patrones se mantienen a lo largo del tiempo.

Palabras clave: Amazonía; murciélagos; influencia climática; influencia lunar, Phyllostomidae.

\section{REFERENCIAS}

Appel, G., López-Baucell, A., Magnusson, W., \& Bobrowiec, P. E. D. (2017). Aerial insectivorous bat activity in relation to moonlight intensity. Mammalian Biology, 85, 37-46.

August, P. V. (1983). The role of habitat complexity and heterogeneity in structuring tropical mammal communities. Ecology, 64, 1495-1507.

Bernard, E. (2002). Diet, activity and reproduction of bats species (Mammalia, Chiroptera) in Central Amazonia, Brasil. Revista Brasileira de Zoologia, 19, 173-188.

Bernard, E. \& Fenton, M. B. (2002). Species diversity of bats (Mammalia: Chiroptera) in forest fragments, primary forests and savannas in central Amazonia, Brazil. Canadian Journal of Zoology, 80, 1124-1140.

Bernard, E., Tavares, V., \& Sampaio, E. (2011). Compilação atualizada das espécies de morcegos (Chiroptera) para a Amazônia Brasileira. Biota Neotropical, 11, $1-12$.

Brigham, R. M. \& Barclay, R. M. R. (1992). Lunar influence on foraging and nesting activity of common poorwills (Phalaenoptilus nuttali). The Auk, 109, 315-320.

Brosset, A. \& Charles-Dominique, P. (1990). The bats of French Guiana: a taxonomic, faunistic and ecological approach. Mammalia, 54, 509-560.

Bobrowiec, P. E., Dos Santos, L., Gazarini, J., \& Haugaasen, T. (2014). Phyllostomid bat assemblage structure in Amazonian flooded and unflooded forest. Biotropica, 46, 312-321.

Bonnacorso, F. J. (1979). Foraging and reproductive ecology in a panamanian bat community. Bulletin of the Florida Museum of Natural History. Biological Sciences, 24, 359-408.
Börk, K. S. (2006). Lunar phobia in the greater fishing bat Noctilio leporinus (Chiroptera, Noctilionidae). Revista de Biología Tropical, 54, 1117-1123.

Bu, Y., Wang, Y., Zhang, Ch., Liu, W., Zhou, H., Yu, Y., \& Niu, H. (2015). Geographical distribution, roost selection and conservation state of cave-dwelling bats in China. Mammalia, 79, 409-417.

Colwell, R. K. \& Coddington, J. A. (1994). Estimating terrestrial biodiversity through extrapolation. Philosophical Transactions: Biological Sciences, 345, 101-118.

Clarke, J. A. (1983). Moonlight's influence on predator/ prey interactions between Short-eared Owls (Asio fiammeus) and deermice (Peromyscus maniculatus). Behavioral Ecology and Sociobiology, 13, 205-209.

Cruz, L. C., Martínez, C., \& Fernandes, F. R. (2007). Comunidades de morcegos em hábitats de uma mata amazônica remanescente na ilha de São Luís, Maranhão. Acta Amazônica, 37, 613-620.

Charles-Dominique, P., Brosset, A., \& Jouard, S. (2001). Atlas dês chauvessouris de Guyane. Patrimoines Naturels, 49, 1-172.

Daly, D. G. \& Prance, G. T. (1989). Brazillian Amazon. In D. G. Campbell, H. D. Hammond (Eds.), Floristic inventory of tropical countries (pp. 401-426). New York, USA: New York Botanical Garden.

Durán, A. \& Canchila, S. (2015). Ensamblaje de murciélagos (Mammalia: Chiroptera) en dos zonas del departamento de Sucre, Colombia. Acta Zoológica Mexicana, 31, 358-366.

Esbérard, C. E. L. (2007). Influência do ciclo lunar na captura de morcegos Phyllostomidae. Iheringia, Séries Zoologicas, 97, 81-85.

Farneda, F. Z., Rocha, R., López-Baucells, A., Sampaio, E. M., Palmeirim, J. M., Bobrowiec, P. E. D., Grelle, C. E., \& Meyer, Ch. (2018). Functional recovery of Amazonian bat assemblages following secondary forest succession. Biological Conservation, 218, 192-199.

Fearnside, P. M. (2014). Impacts of Brazil's Madeira River dams: unlearned lessons for hydroelectric development in Amazonia. Environmental Science \& Policy, $38,164-172$.

Fleming, T. (1986). The structure of neotropical bat communities: a preliminary analysis. Revista Chilena de Historia Natural, 59, 135-150.

Fleming, T., Hooper, E., \& Wilson, D. (1972). Three Central American bat communities: structure, reproductive cycles, and movement patterns. Ecology, 53, 555-569. 
Freeman, P. (2000). Macroevolution in microchiropteran: recoupling morphology and ecology with phylogeny. Evolutionary Ecology Research, 2, 317-335.

Galindo, J. (1998). Dispersión de semillas por murciélagos: su importancia en la conservación y regeneración del bosque tropical. Acta Zoológica Mexicana (n. s.), $73,57-74$.

Gardner, A. L. (2007). Mammals of South America: marsupials, xenarthrans, shrews, and bats (Vol. 1). Chicago, USA: The University of Chicago Press.

Gomes, L. A. C., Pires, A. S., Martins, M. A., Lourenço, E. C., \& Peracchi, A. L. (2015). Species composition and seasonal variation in abundance of Phyllostomidae bats (Chiroptera) in an Atlantic Forest remnant, southeastern Brazil. Mammalia, 79, 61-68.

Gotelli, N. J. \& Colwell, R. K. (2001). Quantifying biodiversity: procedures and pitfalls in the measurement and comparison of species richness. Ecology Letters, 4, 379-391.

Goulding, M., Barthem, R., \& Ferreira, E. (2003). The Smithsonian atlas of the Amazon. Washington, D.C., USA: Smithsonian Institution Press.

Grimshaw, J. R. \& Higgins, C. L. (2017). Environmental correlates of phylogenetic structure in Mexican bat communities. Journal of Mammalogy, 98, 1657-1666.

Gutiérrez, E., Pessoa, V., Aguiar, L. M. S., \& Pessoa, D. M. (2014). Effect of light intensity on food detection in captive great fruit-eating bats, Artibeus lituratus (Chiroptera: Phyllostomidae). Behavioural Processes, 109, 64-69.

Hammer, Ø. \& Harper, D. A. (2016). Paleontological Data Analysis. UK, Oxford: Blackwell Publishing.

Hayes, J. P. (1997). Temporal Variation in Activity of Bats and the Design of Echolocation-Monitoring Studies. Journal of Mammalogy, 78, 514-524.

Heer, K., Helbig-Bonitz, M., Fernandes, R. G., Mello, M A. R., \& Kalko, E. K. V. (2015). Effects of land use on bat diversity in a complex plantation-forest landscape in northeastern Brazil. Journal of Mammalogy, 96, 720-73.

Hock, R. J. (1951). The metabolic rates and body temperatures of bats. Biological Bulletin, 101, 289-299.

Holdridge, L. R. (1967). Life zone Ecology. San José, Costa Rica: Tropical Science Center.

Hoorn, C. \& Wesselingh, F. P. (2010). Amazonia: landscape and species evolution. Oxford: Blackwell Publishing.

Hsieh, T. C., Ma, K. H., \& Chao, A. (2016). iNEXT: An R package for interpolation and extrapolation of species diversity (Hill numbers). Methods in Ecology and Evolution, 7, 1451-1456.
Jones, G., Jacobs, D., Kunz, T., Willigs, M., \& Racey, P. A. (2009). Carpe noctem: the importance of bats as bioindicators. Endangered Species Research, 8, 93-115.

Kalko, E. K. V., Handley, C. O., \& Handley, D. (1996). Organization, diversity and long-term dynamics of a neotropical bat community. In M. L Cody \& J. A. Smallwood (Eds.), Long-term studies of vertebrate communities (pp. 503-553). San Diego, USA: Academic press.

Karlsson, B. L., Eklöf, J., \& Rydell, J. (2002). No lunar phobia in swarming insectivorous bats (family Vespertilionidae). Journal of Zoology, 256, 473-477.

Kunz, T. H. (1982). Ecology of bats. New York, USA: Plenum Press.

Kunz, T. H. \& Fenton, M. B. (2003). Bat ecology. Chicago, USA: The University of Chicago Press.

Lacki, M. J. (1984). Temperature and humidity-induced shifts in the flight activity of little brown bats. Ohio Journal Science, 84, 264-266.

Lang, A. B., Kalko, E. K. V., Rome, H., Bockholdt, C., \& Dechmann, D. (2006). Activity levels of bats and katydids in relation to the lunar cycle. Oecologia, 146, 659-666.

Legendre, P. \& Legendre, L. (2012). Numerical Ecology. Oxford: Elsevier.

Lim, B. K. \& Engstrom, M. D. (2001). Species diversity of bats (Mammalia: Chiroptera) in Iwokrama Forest, Guyana, and the Guianan subregion: implications for conservation. Biodiversity and Conservation, 10, 613-657.

Mello, M. A. R., Schittini, G. M., Selig, P., \& Bergallo, H. G. (2004). A test of the effect of climate and fruiting of Piper species (Piperaceae) on reproductive patterns of the bat Carollia perspicillata (Phyllostomidae). Acta Chiropterologica, 62, 309-318.

Moreno, C. E. (2001). Métodos para medir la biodiversidad. Zaragoza: M\&T-Manuales y Tesis SEA.

Moreno, C. E., Barragán, F., Pineda, E., \& Pavón, M. (2011). Re-análisis de la diversidad alfa: alternativas para interpretar y comparar información sobre comunidades ecológicas. Revista Mexicana de Biodiversidad, 82, 1249-1261.

Moreno, C. E. \& Halffter, G. (2000). Assessing the completeness of bat biodiversity inventories using species accumulation curves. Journal of Applied Ecology, 37, 149-158.

Myster, R. W. (2009). Plant communities of western Amazonia. Botanical Review, 75, 271-291. 
Morrison, D. W. (1978). Foraging ecology and energetics of the frugivorous bat Artibeus jamaicensis. Ecology, 59, 716-723.

Navara, K. J. \& Nelson, R. J. (2007). The dark side of light at night: physiological, epidemiological, and ecological consequences. Journal of Pineal Research, $43,215-224$

Paglia, A. P., Fonseca, G. A. B., Rylands, A. B., Herrmann, G., Aguiar, L. M. S., Chiarello, A. G., Leite, Y. L. R., Costa, L. P., Siciliano, S., Kierulff, M. C., Mendes, S. L., Tavares, V. C., Mittermeier, R. A., \& Patton, J. L. (2012). Annonated checklist of brazilian mammals. Occasional Papers in Conservation Biology, 6, 1-83.

Pereira, M. J., Marquez, J. T., Santana, J., Santos, C. D., Valsecchi, J., Lima, H., Beja, P., \& Palmeirin, J. M. (2009). Structuring of Amazonian bat assemblages: the roles of flooding patterns and floodwater nutrient load. Journal of Animal Ecology, 78, 1163-1171.

Pereira, M. J., Marques, J. T., \& Palmeirin, J. M. (2010) Ecological responses of frugivorous bats to seasonal fluctuations in fruit availability in Amazonian forest. Biotropica, 42, 680-687.

Perigolo, N. A., Medeiros, M. B., \& Simon, M. F. (2017). Vegetation types of the upper Madeira River in Rondônia, Brazil. Brittonia, 69, 423-446.

RADAMBRASIL. (1978). Levantamento de recursos naturais vol. 16: Folha SC.20 Porto Velho. Rio de Janeiro, Brasil: Ministério de Minas e Energia, Departamento Nacional da Producão Mineral.

R Core Team. (2014). R: a language and environment for statistical computing. R Austria: Foundation for Statistical Computing. Retrieved from http://www.rproject.org.

Robinet, C. \& Roques, A. (2010). Direct impacts of recent climate warming on insect populations. Integrative Zoology, 5, 132-142.

Rocha, R., Ovaskainen, O., López-Baucells, A., Farneda, F. Z., Ferreira, D. F., Bobrowiec, P. E. D., Cabeza, M., Palmeirim, J. M., \& Meyer, Ch. (2017). Design matters: an evaluation of the impact of small man-made forest clearings on tropical bats using a before-aftercontrol-impact design. Forest Ecology and Management, 401, 8-16.

Rojas, D., Vale, A., Ferrero, V., \& Navarro, L. (2011). When did plants become important to leaf-nosed bats? Diversification of feeding habits in the family Phyllostomidae. Molecular Ecology, 20, 2217-2228.

Rughetti, M. \& Toffoli, R. (2014). Sex-specific seasonal change in body mass in two species of vespertilionid bats. Acta Chiropterologica, 16, 149-155.

Saldaña-Vásquez, R. A., \& Munguía-Rosas, M. A. (2013). Lunar phobia in bats and its ecological correlates: A meta-analysis. Mammalian Biology, 78, 216-219.

Simmons, N. B. (1996). A new species of Micronycteris (Chiroptera: Phyllostomidae) from northeastern Brazil, with comments on phylogenetic relationships. American Museum of Novitates, 3158, 1-34.

Simmons, N. B. (2005). Order Chiroptera. In D. E. Wilson \& D. Reeder (Eds.), Mammals species of the World: a taxonomic and geographic reference (pp. 312-529). Baltimore. USA: The Johns Hopkins University Press.

Simmons, N. B. \& Voss, S. (1998). The mammals of Paracou, French Guiana: a neotropical lowland rainforest fauna, part 1, Bats. Bulletin of the American Museum of Natural History, 237, 1-219.

Stevens, R. D. \& Willig, M. R. (2002). Geographical ecology at the community level: perspectives on the diversity of new world bats. Ecology, 83, 545-560.

Stoner, K. (2005). Phyllostomid Bat Community Structure and Abundance in Two Contrasting Tropical Dry Forests. Biotropica, 37, 591-599.

Thies, W., Kalko, E., \& Schnitzler, H. (2006). Influence of environment and resource availability on activity patterns of Carollia castanea (Phyllostomidae) in Panama. Journal of Mammalogy, 87, 331-338.

Veloso, H. P., Rangel, A. L. R., \& Lima, J. C. (1991). Classificação da vegetação brasileira, adaptada a um sistema universal. Rio de Janeiro, Brasil: IBGE.

Venables, W. N. \& Ripley, B. D. (2002). Modern applied statistic with S. Four Edition. USA, New York: Springer.

Verde, R. S., Silva, R. C., \& Calouro, A. M. (2018). Activity patterns of frugivorous phyllostomid bats in an urban fragment in southwest Amazonia, Brazil. Iheringia Série Zoologia, 108, 1-7.

Walker, J. (1999). Moontool for Windows. Recovered from http://www.fourmilab.ch/moontoolw. 
Williams, C. B. \& Singh, B. P. (1951). Effect of moonlight on insect activity. Nature, 167, 853-853.

Wolcott, T. G. \& Wolcott, D. L. (1982). Larval loss and spawning behaviour in the land crab Gecarcinus lateralis (Freminville). Journal Crustacean of Biology, $4,477-485$.

Wood, S. (2014). Stable and efficient multiple smoothing parameter estimation for generalized additive models. Journal of the American Statistical Association, 99, 673-686.
Zar, J. H. (1996). Biostatistical Analysis. New Jersey, USA: Prentice Hall.

Zarazúa-Carvajal, M., Saldaña-Vázquez, R. A., SandovalRuiz, C. A., Stoner, K. E., \& Benítez-Malvido, J. (2016). The specificity of host-bat fly interaction networks across vegetation and seasonal variation. Parasitology Research, 115, 4037-4044.

Zur, A. F., Ieno, E. N., Walker, N. J., Saveliev, A. A., \& Smith, G. M. (2009). Mixed effects models and extensions in ecology with $R$. New York, USA: Springer.

See Digital Appendix at: / Ver Apéndice digital en:

revistas.ucr.ac.cr 